\title{
Effets biologiques des neutrons : mécanismes et applications
}

\author{
Ph. VOISIN*
}

(Manuscrit reçule 15 janvier 1999, accepté le 19 juillet 1999)

RÉSUMÉ L'intérêt d'étudier les effets des rayonnements ionisants sur les structures biologiques relève non seulement de la compréhension fondamentale des mécanismes aboutissant aux dommages radio-induits mais aussi de problèmes beaucoup plus pragmatiques que sont les irradiations accidentelles ou la radiothérapie. Parmi l'ensemble de ces êtudes autant fondamentales qu'appliquées, les neutrons occupent une place importante, en raison de leur mode particulier d'ionisation indirecte et de leurs applications tant civiles que militaires. Cette revue a pour but de rappeler certains effets biologiques spécifiques des neutrons et les moyens biologiques de les mesurer. Ces effets sont globalement plus graves que ceux provoqués par les rayonnements de plus faible TEL (rayonnements $X$ et $\gamma$ ) pris comme référence, à tous les niveaux de mesure utilisés, que ce soit les aberrations chromosomiques, la survie cellulaire ou la carcinogénèse. Cette différence est vraisemblablement liée à la densité du dépôt d'énergie dans les cibles vitales de la cellule, et à l'absence de variations significatives liées à l'oxygénation, au débit de dose ou au fractionnement. De tels effets délétères à considérer en cas d'accident de criticité peuvent paradoxalement devenir des avantages en cas d'irradiation thérapeutique.

ABSTRACT Biological effects of neutrons, mechanisms and applications.

The interest to study the ionizing radiations effects on the biological structures concern not only the fundamental comprehension of the mechanisms leading to the radiation damage but also much more pragmatic problems such as the accidental overexposure or radiotherapy treatment. Among these fundamental or applied studies, the neutrons effects take an important part, because of their particular mode of indirect ionization effect and their applications, as well civil as military ones. The purpose of this review is to point out some specific biological effects of neutrons and to describe biological methods to measure them. It clearly appears that neutrons biological effects are more deleterious than those caused by the radiations of lower TEL ( $\mathrm{X}$ - and $\gamma$-rays) taken as reference, for all the measurement levels used, genes mutations, chromosome aberrations, cellular survival or carcinogenesis. This difference is probably related to the density of the energy deposit in the vital cell targets, and to the absence of significant variations related to oxygenation, dose rate or dose fractionation. Such toxic effects, when considered in the course of a criticality accident, can paradoxically become an advantage in the follow-up of therapeutic treatment.

\footnotetext{
Institut de protection et de sûreté nucléaire, IPSN, BP6, F-92265 Fonlenay-aux-Roses Cedex, France. Mél: philippe.voisin@ipsn.fr.
} 
Ph. VOISIN

\section{Introduction}

Les organismes vivants sont généralement très sensibles à l'action des rayonnements ionisants. Pourtant, à considérer l'aspect purement physique, pour une dose létale à l'organisme humain de 10 Gray, délivrée en un temps très court et uniformément, les dommages dus à l'effet direct des radiations (décomposition de l'eau) sont incapables à eux seuls d'expliquer cet effet létal (Poretti, 1988). En réalité, cette sensibilité est due à l'impact des rayonnements sur les structures hautement organisées qui les composent ( $A D N$, membranes), et dont les dommages deviennent rapidement létaux, sinon pour l'organisme entier, du moins pour les cellules qui les subissent.

L'intérêt d'étudier les effets biologiques des rayonnements ionisants relève non seulement de la compréhension fondamentale des mécanismes aboutissant aux dommages radio-induits mais aussi de problèmes beaucoup plus pragmatiques que sont les irradiations accidentelles ou la radiothérapie. Pour les irradiations accidentelles, en effet, il s'agit d'estimer au mieux les doses reçues par les personnes irradiées, la zone irradiée et son étendue. Pour la radiothérapie, il s'agit d'évaluer plus spécifiquement les effets des rayonnements ionisants sur les tissus sains et d'assurer le contrôle des tumeurs, c'est-à-dire de la perte de leur capacité de prolifération. C'est la « mort différée $\gg$ au sens radiobiologique du terme (Tubiana et al., 1986).

Parmi l'ensemble des rayonnements directement et indirectement ionisants, les neutrons occupent une place à part. Le choc direct de la particule neutre peut créer des dommages moléculaires, mais ce sont surtout les protons de recul libérés par diffusion qui sont responsables d'ionisations secondaires et d'un dépôt d'énergie concentré autour du trajet des particules. De ce phénomène a résulté des applications tant civiles que militaires nécessitant des recherches allant de la physique à la clinique en passant par la radioprotection (Barendsen, 1997).

Dans cet article, il est évidemment impossible d'aborder tous ces aspects. Cependant, étudier les effets biologiques des neutrons revient à rappeler certains effets biologiques communs à tous les rayonnements ionisants, et notamment à ceux de faible TEL qui serviront de référence. On pourra ainsi mieux comprendre les particularités des effets neutroniques et les moyens biologiques de les mesurer.

\section{Dommages induits par les rayonnements ionisants au niveau moléculaire}

\subsection{Lésions directes et indirectes de l'ADN}

Lorsqu'un rayonnement frappe une cellule, il a deux points d'impact essentiels : la membrane cytoplasmique et le noyau. Tout le noyau n'est pas nécessairement concerné 
par ce dépôt d'énergie. Ce sont surtout les filaments d'acide désoxyribonucléique ou ADN, supports de l'information génétique de la cellule et de l'organisme, qui sont intégrés dans la structure hautement organisée qu'est le chromosome. Il apparaît clairement que toute modification non contrôlée apportée à l'ADN peut avoir des conséquences dramatiques, soit en affectant la survie de la cellule elle-même, soit en introduisant des mutations dans le message génétique transmis au gré des divisions successives.

Le large spectre des dommages à l'ADN dus aux rayonnements ionisants est la conséquence finale de phénomènes physiques, chimiques et biologiques, se produisant suivant deux mécanismes principaux :

- des dépôts d'énergie sur le complexe macromoléculaire ADN-protéines (effet « direct »);

- la radiolyse de quelques molécules d'eau très proches, que l'on peut assimiler à un cylindre d'hydratation de 2-3 nanomètres d'épaisseur autour de l'ADN (effet « indirect ») (Poretti, 1988), qui, induisant la formation de radicaux libres $\left(\mathrm{H}_{2} \mathrm{O}_{.}^{+}\right.$, e., HO.), pourront, à leur tour, provoquer des lésions du complexe ADN-protéines.

Pour l'exemple, la figure 1 montre les effets théoriques (simulés par méthode de Monte-Carlo) du passage d'une particule de faible TEL sur une cible de taille comparable à l'ADN, par rapport aux dommages provoqués par une particule ionisante de fort TEL, disons un proton secondaire au passage d'un neutron. On comprend aisément que l'une des conséquences principales du passage d'une particule de TEL élevé sera l'initiation de nombreux dommages à l'ADN dans un espace restreint, dommages qui, de ce fait, seront d'autant plus difficilement réparables.

Ces dommages peuvent être succinctement classés en lésions simple brin, lésions doubles brins et altérations des bases (Fig. 2). Si la lésion simple brin est la rupture d'une hélice d'ADN, la lésion double brins est la dégradation séparée des deux chaînes de l'ADN, à une distance ne dépassant pas quelques nucléotides. Elle peut être produite, soit par la même particule d'énergie suffisante pour créer les deux lésions successivement, soit par deux particules passant l'une après l'autre dans un délai suffisamment court pour que la deuxième lésion se produise avant que la première ait été réparée. En outre, différentes sortes de pontage (cross links) sont également mis en évidence : liaisons covalentes au sein d'une même chaîne ou entre les deux brins de l'hélice ; liaisons covalentes ADN-protéines de liaison; formation de dimères entre deux bases adjacentes.

Les fréquences relatives des principales lésions créées par des rayonnements ionisants de faible TEL sont rassemblées dans le tableau I, tous effets confondus. Le nombre de lésions simple brin et doubles brins croît linéairement avec la dose pour tous les TEL étudiés, quelle que soit la technique analytique utilisée (Lett, 1992). Cependant, 


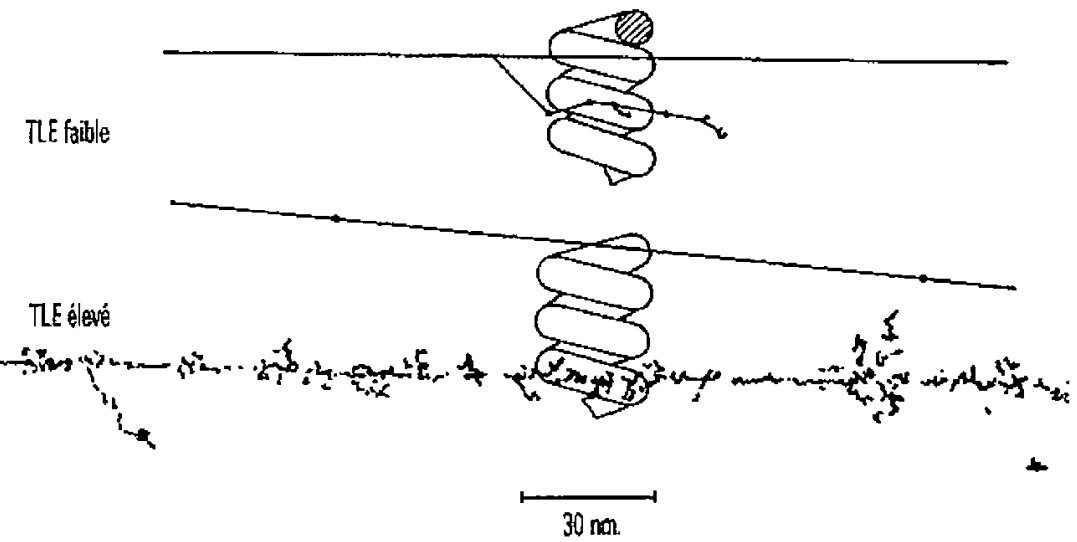

Fig. 1. - Représentation schématique de la distribution des ionisations sur le passage d'une particule de faible TEL (en haut) et de TEL élevé (proton secondaire à un flux de neutron, par exemple, en bas), traversant une molécule d'ADN. Le passage d'une particule de TEL élevé est à la source de lésions multiples localisées dans un faible volume qui seront d'autant plus difficilement réparables (d'après Galle and Paulin (1992)).

Diagrammatic representation of the ionizations distribution after the passage of a particle of low LET (in top) and of high LET (secondary proton to neutron flux, for example in bottom), through a DNA macromolecule. The passage of a high LET particle induces multiple lesions, localised in a small volume, which are not easily reparable (according to Galle and Paulin (1992)).

des différences qualitatives et quantitatives dans la formation des lésions doubles brins existent quand le TEL augmente (par exemple entre rayons $\gamma$ et neutrons de fission). Ceci s'expliquerait par la diminution de la capacité de la cellule à réparer les cassures de l'ADN, que ce soit en condition d'aérobie ou d'hypoxie. Le rôle de la colonne d'hydratation a été reconsidéré récemment (Geacintov and Swenberg, 1991). À l'aide de substances captant les radicaux libres («scavengers»), il a été établi que les radicaux hydroxyle ne pouvaient attaquer directement l'ADN que s'ils se formaient à 2-3 nm seulement. De plus, on a pu montrer qu'une fois initié, ce type de dommage ne pouvait être stoppé. Finalement, pour une irradiation de 1 Gy qui devrait produire 1000 lésions simple brin dans la cellule, $35 \%$ résulteraient de cet effet dit indirect.

Il est vraisemblable que la coupure franche des deux chaînes ne soit pas le seul phénomène responsable de la formation de lésions double brins. En effet, on sait que les systèmes de réparation de l'ADN sont très sensibles à l'état de la chaîne autour de n'importe quelle lésion. Un simple dommage aux bases mal réparé pourrait aussi se transformer en rupture simple brin (Preston, 1990). 


\section{DÉTÉRIORATION DES FILAMENTS D'ADN}

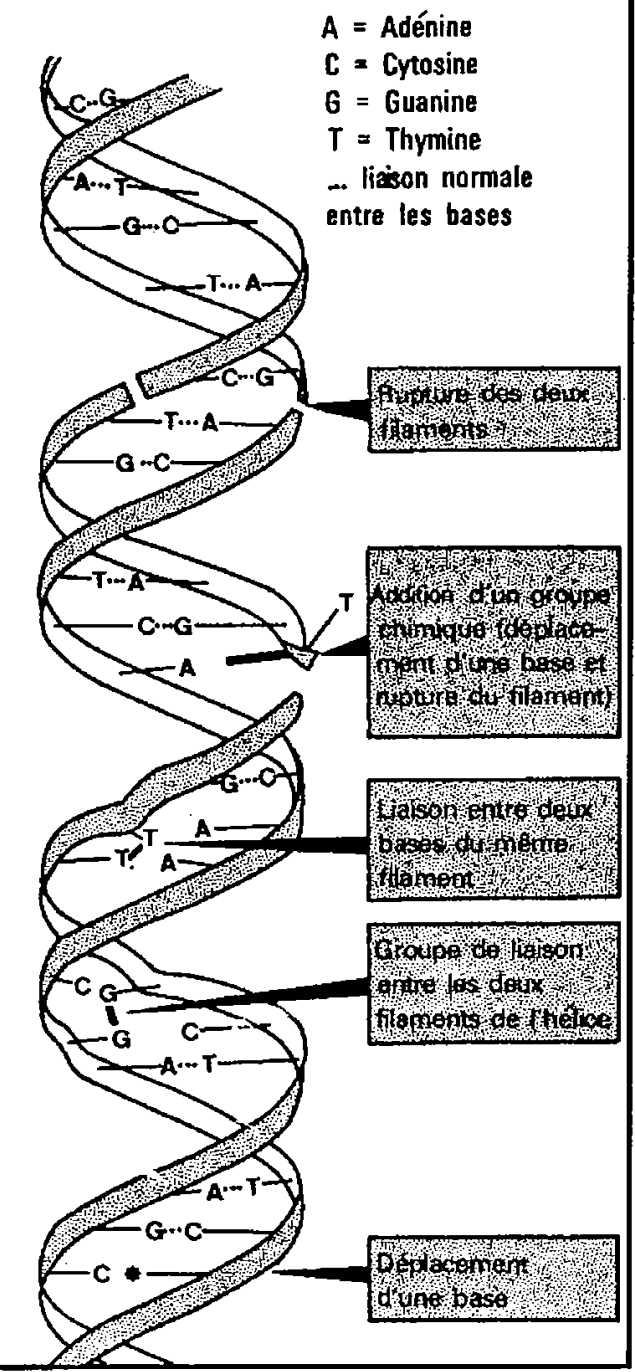

Fig. 2. - Les différentes types de lésions de l'ADN produites par les rayonnements directement et indirectement ionisants (d'après Poretti (1988)).

Types of DNA lesions produced by directly and indirectly ionizing radiations (according to Poretti (1988)). 
Ph. VOISIN

\section{TABLEAU 1}

Fréquence relative des principales lésions de l'ADN créées dans une cellule par une dose absorbée de $1 \mathrm{~Gy}$ due à des rayonnements ionisants de faible TEL

(RX ou $\gamma$ ) (d'après Galle and Paulin (1992)).

Relative yield of the main DNA lesions induced by a 1 Gy absorbed dose of sparsely ionizing radiation (RX ou $\gamma$ ) (according to Galle and Paulin (1992)).

\begin{tabular}{|c|c|}
\hline Mamne des lésions de l'ADN & NombredCeliulolay \\
lésions simple brin & $500-1000$ \\
lésions double brinss & 40 \\
dommages des bases & $800-2000$ \\
dommages des sucres & $800-1600$ \\
Pontages ADN - ADN & 30 \\
Pontages ADN - protéines & 150 \\
\hline
\end{tabular}

\subsection{Mécanismes de réparation de l'ADN}

La conservation du matériel génétique demande non seulement un mécanisme extrêmement précis de copie des séquences de l'ADN avant qu'une cellule ne se divise, mais aussi un mécanisme de réparation des nombreuses lésions accidentelles qui se produisent continuellement. La plupart des modifications spontanées sont immédiatement effacées par un processus de correction. Exceptionnellement, ce processus de correction fait défaut et permet une modification permanente de l'ADN.

Le mécanisme de base de la réparation de l'ADN implique trois étapes (Albert et al., 1990) (Fig. 3) :

1) la partie modifiée d'une chaîne d'ADN est reconnue et éliminée par des endonucléases, laissant une brèche dans l'ADN. C'est l'excision ;

2) l'ADN polymérase se fixe à l'extrémité de la chaîne d'ADN coupée et comble la brèche nucléotide après nucléotide en reconstituant l'information originale à partir de la chaîne non modifiée. C'est la resynthèse ;

3) lorsque l'ADN polymérase a comblé la brèche, cette dernière est soudée par une ADN ligase, qui complète le processus de reconstruction.

Dans le cas des lésions simple brin et de la majorité des dommages aux bases, la réparation est systématiquement fidèle. Dans le cas des lésions double brins ou lorsque le dommage est important (cas des neutrons de fission), la réparation peut ne pas être complète et être à l'origine de lésions définitives. Le dommage peut aussi 

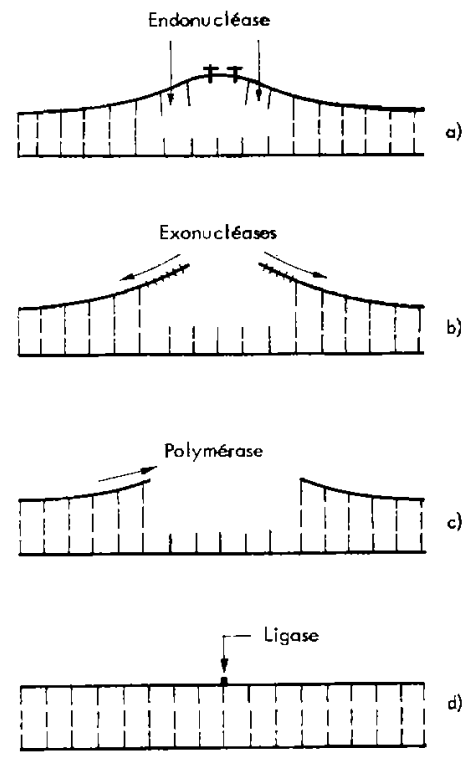

Fig. 3. - Schéma type de la réparation de l'ADN par excision-resynthèse.

Diagram of the DNA repair by excision-resynthesis mechanism.

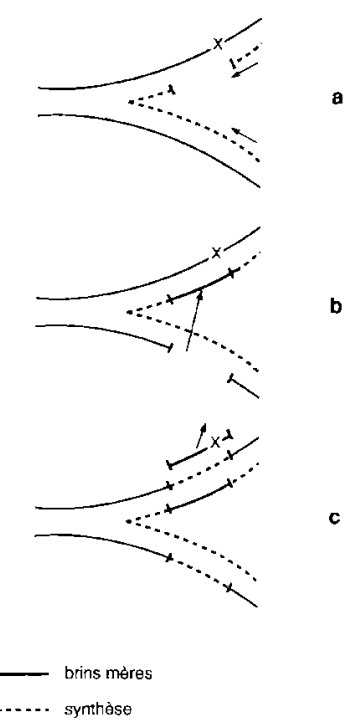

Fig. 4. - Schéma-type de la réparation de l'ADN par recombinaison post-réplicative.

Diagram of the DNA repair mechanism by post-replicative recombination. 
se produire sur une cellule en phase $S$. Un mécanisme de réparation, appelé recombinaison post-réplicative, entre en jeu (Fig. 4). L'ADN-polymérase ne peut dupliquer une structure endommagée et donc va laisser une brèche sur l'hélice-fille. Un échange se produit alors entre cette brèche et la partie intacte correspondante de l'hélice-mère. Puis cette nouvelle brèche est comblée par l'ADN polymérase qui prend comme modèle l'autre portion intacte de l'hélice-fille. Un processus similaire, quoique plus complexe, pourrait être à l'origine de la réparation des lésions double brins chez les bactéries et les eucaryotes. Finalement, la cellule a développé des mécanismes permettant de réparer des fragments entiers d'ADN en réponse à d'importantes lésions (réponse S.O.S.) pour lesquelles l'intégrité de l'ADN n'est plus respectée.

\subsection{Lésions de l'ADN et neutrons}

Pour mieux comprendre les effets de différents rayonnements sur les lésions de l'ADN, des études ont été effectuées en utilisant un antimétabolite de l'ADN, la Cytosine arabinoside (Ara C), sur les lymphocytes (Preston, 1990). L'Ara C paraît inhiber sélectivement la réparation des dommages aux bases sans affecter la réparation des ruptures simple et double brins. Si les lymphocytes sont irradiés par un rayonnement $\mathrm{X}$ et incubés avec de l'Ara $C$ durant 1, 2 ou 3 heures après irradiation, la fréquence des aberrations chromosomiques induites dans ces cellules est considérablement augmentée par rapport à la fréquence observée dans les cellules irradiées sans post-traitement par l'Ara C. De plus, la fréquence des aberrations augmente avec le temps d'incubation. Par contre, si les lymphocytes sont exposés à des neutrons de fission, puis incubés avec de l'Ara C, la fréquence des aberrations n'est pas significativement différente de celle obtenue à partir de cellules irradiées seulement. Deux conclusions s'imposent. D'une part, la fréquence de lésions double brins semble beaucoup plus importante dans l'ADN exposé aux neutrons par rapport aux rayonnements de plus faible TEL, et donc la probabilité pour ces lésions double brins de produire des remaniements (aberrations chromosomiques, mutations) est également plus importante. D'autre part, on peut admettre des différences dans les mécanismes par lesquels les neutrons et les RX induisent des aberrations chromosomiques. Les dommages aux bases nucléiques sont un constituant majeur de l'induction des aberrations par les RX, tandis que les lésions double brins auraient une importance plus grande dans la production des aberrations chromosomiques par les neutrons.

\subsection{Effets des rayonnements sur les chromosomes}

L'effet des rayonnements ionisants au niveau de la double hélice d'ADN se traduit sous forme de lésions primaires. Les zones lésées sont la plupart du temps restaurées, redonnant un chromosome apparemment normal. Un échec de restauration peut cependant apparaître. D'autres fois, se produit un échange entre un et plusieurs chromosomes. Ce remaniement a un aspect dépendant du nombre de lésions primaires 
en cause, de leur situation, de la cinétique de réparation et de l'organisation du génome à l'intérieur du noyau (AIEA, 1986; Bender et al., 1988; Lloyd, 1990).

Il faut distinguer les lésions se produisant au cours de l'interphase de celles se produisant durant les phases S ou G2 du cycle cellulaire. Dans le premier cas, ce sont des aberrations de type chromosomique, dans lesquelles la lésion a été dupliquée. Dans le deuxième cas, ce sont des aberrations de type chromatidique, une seule chromatide étant lésée sur les deux dupliquées. Nous nous intéressons seulement aux premières, qui sont les plus importantes en dosimétrie biologique (Fig. 5).

- Un échec de restauration peut apparaître sous l'aspect d'un fragment acentrique, le chromosome ayant perdu la partie correspondante portant une délétion. Cependant, cette anomalie est loin d'être spécifique de l'irradiation puisqu'elle peut être observée dans tous les cas de mutagenèse.

- Dans un remaniement interchromosomique, les deux lésions primaires appartiennent à deux chromosomes différents. Selon qu'un seul ou les deux centromères sont impliqués dans la réparation, le chromosome remanié a l'aspect d'une translocation ou d'un dicentrique. La technique de coloration utilisée en cytogénétique conventionnelle ne permet d'observer que les dicentriques. Ces derniers peuvent exister indépendamment d'une irradiation, mais à une fréquence de 1 pour 2000 cellules environ. L'observation des translocations nécessite des techniques de marquage plus sophistiquées (bandes $\mathrm{G}$, hybridation in situ fluorescente)

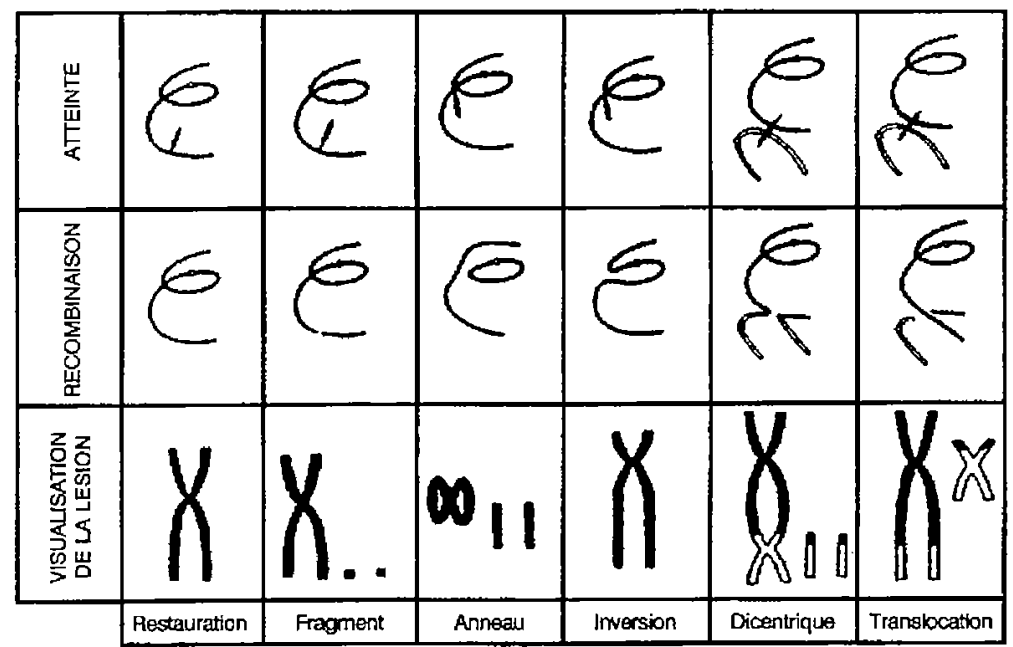

Fig. 5. - Nature et formation des aberrations chromosomiques stables et instables radio-induites (d'après Doloy, 1991).

Various types and formation mechanisms of radio-induced stable and unstable chromosome aberrations (from Doloy, 1991). 
- Dans un remaniement intrachromosomique, les deux lésions primaires appartiennent au même chromosome. Le chromosome remanié prend alors l'aspect soit d'une inversion (réunion de deux portions du chromosome, normalement non contiguës), soit d'un anneau centrique (réunion des deux extrémités d'une même portion chromosomique). Seuls les anneaux centriques peuvent être observés par les techniques de coloration classiques. La fréquence d'observation d'un anneau centrique, sans qu'il y ait eu d'irradiation, est de 10 fois inférieure à celle d'un dicentrique. Pour observer les inversions, on peut utiliser les mêmes techniques que pour les translocations.

\subsection{Aberrations chromosomiques et relations dose-effet}

La fréquence des aberrations chromosomiques, est liée quantitativement à la dose par l'intermédiaire d'une fonction mathématique de forme générale :

$$
Y=c+a D+B D^{2} \quad(\text { Edwards, 1990) }
$$

Avec $Y=$ nombre d'aberrations parmi les cellules observées ;

$D=$ dose physique (en gray) ;

$c=$ fréquence des aberrations sans irradiation ;

$a=$ coefficient linéaire de la dose ;

$b=$ coefficient quadratique de la dose.

Pour les rayonnements de faible TEL, cette relation dose-effet est habituellement décrite par une régression linéaire-quadratique (Fig. 6). La pente initiale de la courbe est d'autant plus faible que le débit de dose est réduit. Par contre, pour les neutrons ou plus généralement les rayonnements de TEL élevé, la relation tend à être linéaire. De plus, la pente est plus élevée que celle des rayonnements $\mathrm{X}$ et $\gamma$, traduisant une production d'aberrations plus importante. Le nombre d'aberrations paraît alors directement proportionnel à la dose, sans effet notable du débit de dose. Dans le cas d'un accident de criticité, où le flux est généralement mixte (neutrons $+\gamma$ ), on voit que pour la même dose, les effets biologiques dus aux neutrons sont prédominants sur ceux dues aux rayons $\gamma$. La connaissance de ces effets relatifs peut se révéler essentiel dans le traitement d'éventuels accidents d'irradiation.

On a tenté de relier l'équation de la courbe à une interprétation physiologique de la formation des aberrations chromosomiques. Reprenant le même modèle expérimental, Preston (1990) a irradié des lymphocytes en interphase par des doses de RX entre 0,5 et 3 Gy puis incubés avec de l'Ara $C$ durant 1 à 4 heures. La fréquence d'aberrations radio-induites augmente logiquement avec la dose. De 


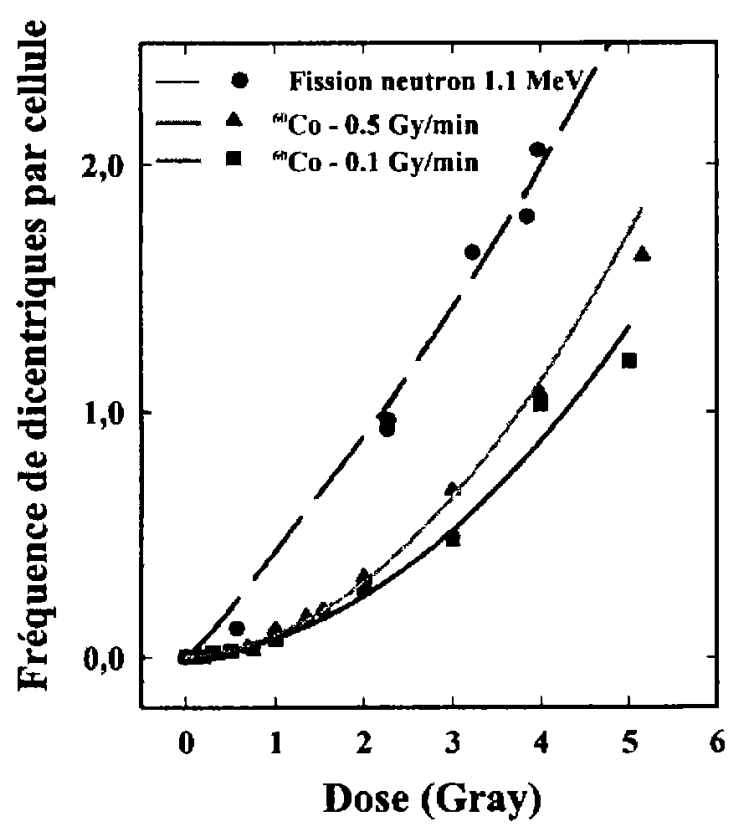

Fig. 6. - Relations dose-effet établies en dénombrant les aberrations chromosomiques induites dans les lymphocytes du sang périphérique par des rayonnements ionisants de différentes qualités et à différents débits de dose.

Dose-effect relationships fitted by scoring unstable chromosome aberrations induced in blood peripheral lymphocytes by ionizing radiation of different qualities and of various dose rates.

plus, à dose constante, la fréquence d'aberrations augmente aussi avec la durée d'incubation à l'Ara C. Puisque cette pente est une mesure du taux de lésions converties en aberrations sans pouvoir être réparées, le taux de réparation augmente donc avec la dose. Compte-tenu de l'action de l'Ara C, une part de ces aberrations est due à la non réparation ou à la mauvaise réparation des dommages aux bases. Par contre, ce phénomène n'est pas observé pour une dose de 0,5 Gy, ce qui permet de déduire que, pour des doses faibles, seules les doubles cassures de l'ADN sont impliquées dans la formation des aberrations chromosomiques.

Partant de ces hypothèses pour un rayonnement de faible TEL, on peut s'attendre à ce que la formation des aberrations formant la composante linéaire d'une relation dose-effet soit due à la mauvaise réparation de lésions double brins. De plus, les deux composantes de la partie quadratique de la courbe pourraient être la somme des deux types de lésions majeures à l'ADN, à savoir les lésions double brins et les dommages aux bases. Inversement, lorsqu'on examine les relations dose-effet dues aux neutrons, qui apparaissent linéaires pour la plupart, la grande 
majorité des aberrations ainsi produites seraient dues à la mauvaise réparation des lésions double brins.

Il reste à considérer l'influence du débit de dose dans la production des aberrations chromosomiques. Quand des cellules sont exposées aux RX à un faible débit de dose (c'est-à-dire avec une dose totale délivrée sur plusieurs heures), la fréquence d'aberrations observées est plus faible que celle obtenue quand la dose est délivrée en quelques instants (secondes, minutes). Le niveau de réduction varie avec la dose, car s'il est important pour les fortes doses, il est pratiquement inexistant pour les doses les plus faibles ( $0,5 \mathrm{~Gy}$ et au-dessous). La courbe ainsi obtenue est linéaire et sa pente est équivalente à la composante linéaire de la relation linéaire-quadratique obtenue en cas d'irradiation aiguë. Ceci semble lié aux deux phénomènes simultanés de production et de réparation des lésions. Intuitivement on comprend que, en raison du laps de temps important qui sépare deux coupures simples de l'ADN et qui permet la réparation, seules les doubles lésions créées simultanément ont quelque chance de produire des aberrations. Ceci est similaire aux mécanismes de production des aberrations aux faibles doses. Pour ce qui est des neutrons, la production d'aberrations apparaît largement indépendante du débit de dose, ce qui est en accord avec l'hypothèse d'une production quasi exclusive de lésions double brins.

On voit ici l'intérêt d'utiliser le dénombrement des aberrations chromosomiques radio-induites dans les lymphocytes du sang périphérique lors d'une suspicion d'exposition aux rayonnements ionisants ou d'atteinte accidentelle avérée, où il est particulièrement important de définir au mieux la dose reçue, la zone corporelle atteinte et son étendue. Ce type de dosimétrie biologique constitue actuellement l'indicateur biologique le plus spécifique des rayonnements ionisants.

\subsection{Dosimétrie biologique et accident de criticité}

Lors d'un accident de criticité, il y émission d'un flux mixte neutrons $+\gamma$. Selon la configuration accidentelle, le rapport de ces deux rayonnements est différent, mais nous avons montré que l'effet des neutrons sur les aberrations chromosomiques, et donc sur l'organisme, est nettement plus important que celui des rayonnements $\gamma$. Une méthode de calcul particulière peut être utilisée (AIEA, 1986) pour quantifier les parts respectives de neutrons et de rayonnement $\gamma$ présents dans le flux mixte, et par conséquent de situer leurs effets biologiques respectifs. Ainsi, on considère que la fréquence d'aberrations chromosomiques observée dans les lymphocytes du sang périphérique est la somme des aberrations dues au rayonnement $\gamma$ et de ceux dues au neutrons. Les doses respectives sont obtenues en utilisant des relations effets-dose, linéaire pour les neutrons et linéaire-quadratique de pente plus faible pour le rayonnement $\gamma$. La seule information externe indispensable est le rapport global entre les deux types de rayonnements qui est une donnée physique. 
Une vérification expérimentale de cette estimation a été réalisée au cours d'une récente intercomparaison internationale, au cours de laquelle dosimétrie physique et dosimétrie biologique ont été comparées (Voisin et al., 1997). Du sang total était irradié à deux doses dans plusieurs configurations (air libre, au contact d'un mannequin) avec un rapport neutron $/ \gamma$ de 1 et de 5 respectivement. Le tableau II donne la correspondance entre la dose estimée par la biologie pour les composantes $\gamma$ et neutron et la dosimétrie physique prise comme référence. Il met en évidence une estimation très proche de la valeur attendue dans toutes les configurations expérimentales. Ces résultats obtenus sur du sang irradié in vitro constituent une approche de ce qui se passerait in vivo lors d'un véritable accident de criticité. En effet, l'hétérogénéité de dose due à l'atténuation des neutrons par l'hydrogène des tissus peut être compensée en partie par la diffusion des protons de recul et des électrons de haute énergie (Voisin et al., 1997).

\section{Dommages induits par les rayonnements ionisants au niveau cellulaire}

Quelques heures après l'irradiation par plusieurs dizaines de gray, de cellules en culture ou in vivo, on peut observer l'arrêt de toute fonction cellulaire, une lyse des membranes nucléaire et cellulaire. Finalement, la cellule se désagrège et disparaît. Après

\section{TABLEAU II}

Décomposition des effets biologiques dus aux neutrons et au rayonnement $\gamma$ suite à une irradiation in vitro de prélèvements sanguins par un flux mixte neutron $/ \gamma$ simulant un accident de criticité. Le tableau montre l'index neutron et l'index $\gamma$ de l'estimation de dose obtenue par la dosimétrie biologique par cytogénétique conventionnelle, en prenant comme référence les estimations données par la dosimétrie physique (d'après Voisin et al. (1997)).

Decomposition of the biological effects of fission neutrons and $\gamma$ rays after a blood in vitro irradiation by a mixed neutron $/ \gamma$ flux simulating a criticality accident.

The table shows the neutron and $\gamma$ ratios of the dose estimates obtained by conventional cytogenetics to dose estimates given by physical dosimetry like reference term (according to Voisin et al. (1997)).

\begin{tabular}{|c|c|c|c|}
\hline Configuration de ITriadiation & Posithonnement du sang irradié & Index Neutron & Index $\gamma$ \\
\hline Ecran plomb & Air libre & 0,91 & 1,10 \\
\hline (neutrons $/ \gamma \cong \mathbf{5}$ ) & Mannequin & 1,03 & 0,79 \\
\hline Source nue & Air libre & 0,94 & 0,77 \\
\hline (neutrons $/ \gamma \cong 1$ ) & Mannequin & 1,02 & 0,75 \\
\hline
\end{tabular}


une irradiation à des doses moins élevées, les cellules semblent normales tant d'un point de vue morphologique que fonctionnel. La cellule parvient à se diviser sur quelques cycles, mais finit par se dégrader et disparaître. C'est au moment de la mitose que s'expriment généralement les lésions que la cellule n'a pu réparer. Cette séquence létale définit la «mort différée » au sens radiobiologique du terme. Cette mort différée explique certains aspects, apparemment contradictoires, observés après irradiation in vivo des tumeurs ou des tissus sains avoisinants. En effet, lorsque la cellule a créé un « clone » de 50 cellules, elle est considérée comme normale d'un point de vue de la survie. Elle peut toutefois être anormale au plan génétique et avoir franchi le premier stade de la transformation cellulaire (Tubiana et al., 1986).

\subsection{Courbes de survie cellulaire}

L'intérêt de la détermination des courbes de survie dérive non seulement de la nécessité de comprendre les mécanismes des dommages radio-induits, mais aussi d'appréhender les conséquences pour la radiothérapie des tumeurs malignes. Les dommages aux tissus sains aussi bien que le contrôle des tumeurs dépendent de la réponse cellulaire, en particulier du blocage de leur capacité à se reproduire indéfiniment.

Le calcul du taux de survie cellulaire consiste donc globalement à soumettre des cellules à des doses croissantes de rayonnements ionisants, puis à compter le nombre de cellules qui parviendront à se diviser suffisamment pour former un clone de 50 cellules au moins. Plusieurs méthodes ont été expérimentées puis perfectionnées au cours du temps. La première méthode consiste à irradier les cellules in vitro, puis à les cultiver isolément dans un milieu de culture adéquat, enfin à compter le nombre de clones. Mais par cette technique, on ne prend pas en compte ce qui fait la spécificité d'un tissu, à savoir les communications intercellulaires, les échanges de métabolites, la présence d'oxygène, et la disponibilité des facteurs de croissance. On a donc choisi d'irradier la tumeur sur l'animal vivant, puis de tester la viabilité des cellules, soit in vitro comme précédemment, soit en comptant les colonies formées au niveau d'un organe cible (poumon, rate) sur un animal « receveur » après injection de cellules isolées (Tubiana et al., 1986).

Les formes que peuvent revêtir les courbes de survie cellulaire peuvent être classées en deux grandes catégories (Fig. 7). Dans la survie exponentielle, les points expérimentaux sont décrits par une fonction du type $S(D) / S(0)=\exp (-a D)$. Les courbes de survie avec épaulement sont caractérisées aux faibles doses par un épaulement initial commençant avec une pente d'ordre 0 , suivie par une pente exponentielle. Le prolongement de cette pente vers l'axe des abscisses permet de définir le nombre d'extrapolation. Ce nombre varie notablement en fonction des différents types cellulaires et des conditions de culture et sa signification biologique reste ambiguë. C'est pourquoi on préfère décrire les courbes globalement 


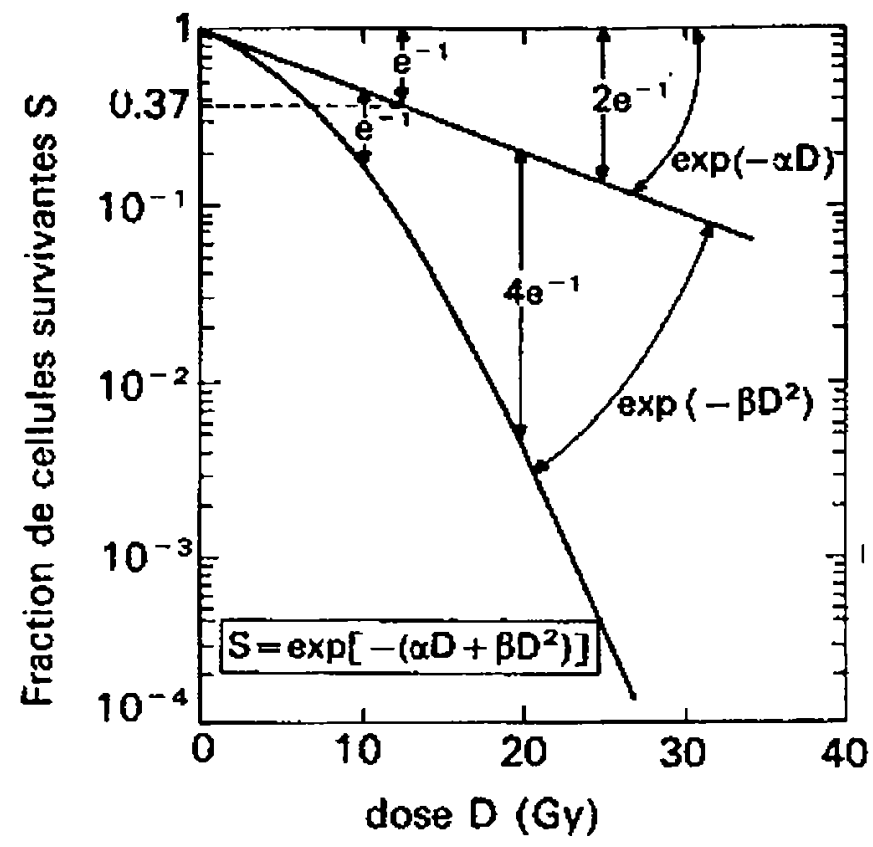

Fig. 7. - Courbes de survie obtenues en exposant des populations cellulaires à des doses croissantes de rayonnements ionisants, puis en comptant le nombre de cellules survivantes ayant formé par division des populations isolées (clones) de plus de 50 cellules (d'après Elkind (1997)).

Survival curves obtained by exposing cell populations to increasing doses of ionizing radiation, then by counting the clones of more than 50 cells, formed by division of the surviving populations cells (according to Elkind (1997)).

par l'association d'une fonction linéaire et d'une fonction quadratique : $S(D) / S(0)$ $=\exp -\left(a D+b D^{2}\right)$, où $a$ et $b$ représentent respectivement les coefficients linéaire et quadratique de la relation logarithmique effet-dose.

Dans la plupart des systèmes cellulaires, la forme de la courbe de survie passe ainsi d'une forme linéaire-quadratique à une forme linéaire (en coordonnées semilogarithmiques) dès lors que le TEL des rayonnements s'accroît, avec une augmentation progressive de la pente initiale, une réduction de l'épaulement et une augmentation de la valeur absolue de la pente aux fortes doses. On sait que l'efficacité maximale des effets biologiques est atteinte pour un TEL d'environ $100 \mathrm{keV} / \mu \mathrm{m}$, que ce soit pour la perte des propriétés clonogéniques ou les dommages chromosomiques engendrés aux cellules. Pour la modélisation, cela se traduit par une augmentation du coefficient linéaire $D$ par rapport au terme quadratique 
$D^{2}$ (Barendsen, 1997). Pour rendre compte de cette radiosensibilité cellulaire, un index caractéristique $D_{0}$ définit la dose nécessaire pour réduire le taux de survie de $37 \%$, et est calculé dans la partie linéaire de la courbe.

Comme pour les lésions de l'ADN et la production d'aberrations chromosomiques, on a essayé de relier les courbes de survie cellulaire à des événements biologiques susceptibles d'expliquer la mort des cellules. On a ainsi suggéré que la mort cellulaire pouvait être la conséquence de lésions létales, sublétales et potentiellement létales. L'importance relative de ces différents types de lésions est inévitablement lié à la nature du rayonnement, éventuellement au débit et au fractionnement de la dose (Barendsen, 1997). Il faut souligner que ces lésions cellulaires ne doivent pas être confondues avec les lésions de l'ADN, même si un parallélisme parfois étroit a été montré entre les deux phénomènes. Les études récentes sur les différents types de mort cellulaire radio-induite, ont révélé que les mécanismes sousjacents devaient être étendus à la membrane cellulaire, aux mitochondries, aux signaux de communications, etc.

Les lésions létales sont des lésions qui entraînent de manière inéluctable la mort de la cellule. Une pente non nulle à l'origine de la courbe de survie peut refléter leur existence aux faibles doses. Dans le modèle linéaire-quadratique, elles seraient représentées par le coefficient linéaire. Les lésions sublétales (SL), par opposition aux précédentes, ne sont pas irréversibles. Dans les conditions normales de la vie cellulaire, $80 \%$ d'entre elles sont généralement réparées en quelques minutes, voire quelques heures. Le taux de réparation est fonction de la localisation des lésions, de la capacité de réparation de la cellule et du temps dont cette dernière dispose pour réparer. La fréquence de ces lésions est représentée dans le modèle linéairequadratique par le coefficient quadratique. Les lésions sublétales sont produites non seulement par les rayonnements de faible TEL, mais aussi par ceux des TEL élevés, pour lesquels les courbes de survie deviennent exponentielles. Ces lésions ne deviennent létales que dans la mesure où elles ne sont pas réparées lorsqu'une lésion supplémentaire est créée à leur niveau. On peut donc penser que les facteurs dose et débit de dose jouent un rôle essentiel dans leur potentialité d'accumulation. Lors d'irradiations fractionnées, c'est-à-dire espacées dans le temps, un taux de survie supérieur peut être observé, car les cellules réparent une partie de leurs lésions entre les deux fractions de dose. L'importance de ces phénomènes de réparation cellulaire peut ainsi être exprimée en terme de «dose additionnelle» nécessaire pour obtenir un taux de survie ou un effet biologique donné. Finalement, les lésions potentiellement létales ont été détectées expérimentalement comme des lésions sujettes à disparition ou expression, selon les conditions auxquelles les cellules sont exposées après irradiation. En maintenant les cellules en phase de repos ou en phase de dépression métabolique après une certaine dose de rayonnements, la réparation des lésions potentiellement létales entraîne une amélioration du taux de prolifération. Ce type de lésion apparaît donc comme un compromis entre les lésions létales et sublétales, puisqu'elles interviendraient à la fois dans la composante linéaire et quadratique des courbes de survie. 


\subsection{Efficacité Biologique Relative (EBR) des neutrons}

L'identification de radiosensibilités cellulaires distinctes face à différents types de rayonnements ionisants nécessite de caractériser l'effet biologique produit à dose absorbée équivalente. L'efficacité biologique relative (EBR) d'un rayonnement à

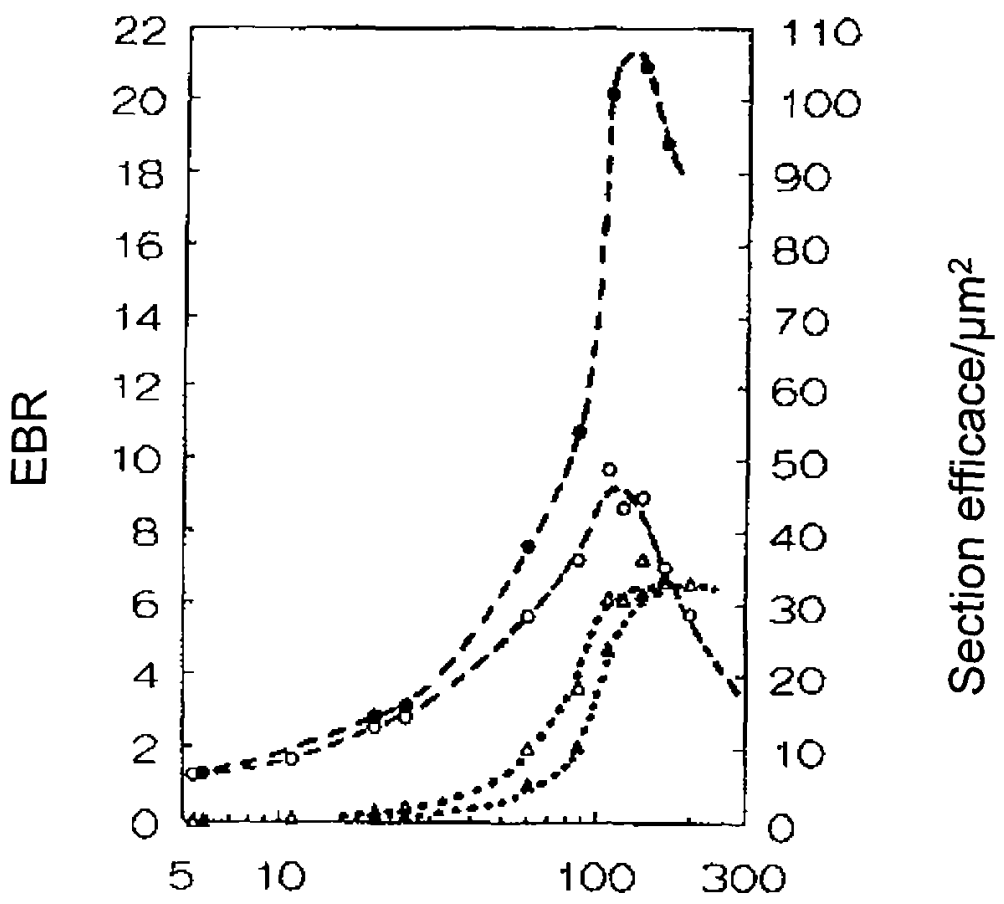

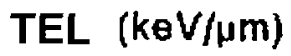

Fig. 8. - Rapport de l'Efficacité Biologique Relative (EBR) au Transfert Linéique d'Energie (TEL) dans la survie de cellules humaines irradiées en conditions normale (O) ou hypoxique (•). Les sections efficaces $(\triangle, \Delta)$ sont calculées à partir d'une courbe de survie linéaire-quadratique correspondant à la dose nécessaire pour infliger une lésion directement létale à chaque cellule (d'après Barendsen (1997)).

Relative Biological efficiency (RBE) according to Linear Energy Transfer (LET) for the survival of human cells irradiated under standard conditions (O) or hypoxic conditions ( $\bullet$ ). The cross sections $(\triangle, \Delta)$, calculated from a linear-quadratic survival curve, correspond to the dose needed to induce a direct lethal lesion to each cell (according to Barendsen (I997)). 
Ph. VOISIN

étudier («test ») par rapport à un rayonnement de référence ( « ref ») est le rapport Dref/Dtest dans lequel Dref et Dtest sont les doses absorbées nécessaires à l'obtention d'un même effet (Figs. 8 et 9). L'EBR n'a de signification que pour un critère biologique donné et un niveau d'effet bien défini, par exemple la survie cellulaire, les aberrations chromosomiques, les mutations géniques. Le rayonnement pris

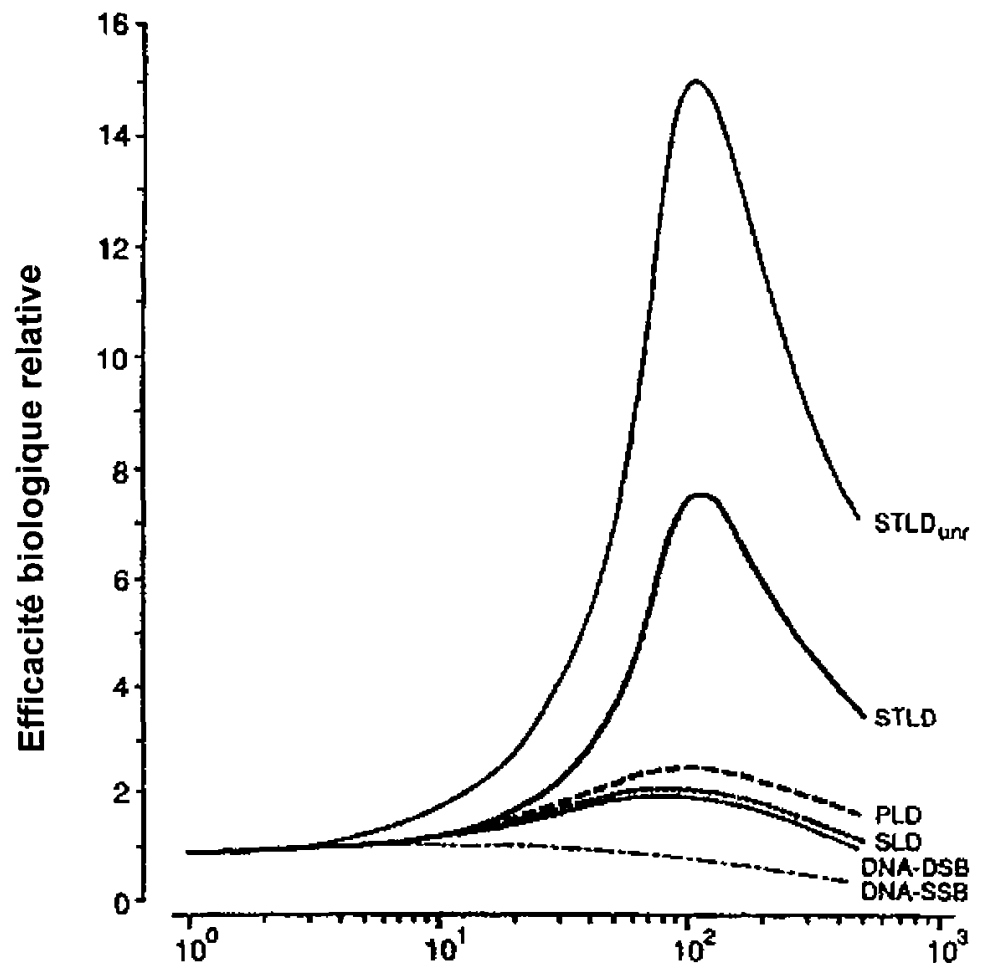

Transfert linéique d 'énergie en $\mathrm{KeV} / \mu \mathrm{m}$ par unité de tissu

Fig. 9. - Représentation schématique de l'importance relative des différents types de lésions létales (STLD), sub-létales (SLD) et potentiellement létales $(P L D)$ dans la mort des cellules de mammifêres, en fonction du rapport EBR/TEL. Sont représentés également en fonction du rapport EBR/TEL l'évolution du nombre de lésions double brins (DNA-dsb) et de lésions simple brin (DNA-ssb) de l'ADN (d'après Barendsen (1997)).

Diagrammatic representation of the respective importance of lethal (STLD), sub-lethal (SLD) and potentially lethal (PLD) DNA lesions for inducing mammals cell death, according to $R B E / L E T$ ratio. Are also represented to $R B E / L E T$ ratio the respective evolution of the DNA double strand (DNA-dsb) and single strand breaks (DNA-ssb) yields (according to Barendsen (1997)). 
comme référence est généralement le rayonnement $\gamma$ du ${ }^{60} \mathrm{Co}$ ou les rayons $\mathrm{X}$ de haute énergie (>1 MeV). L'EBR varie à la fois avec le TEL et la dose absorbée. Ainsi, à dose constante, l'efficacité biologique relative augmente avec le TEL jusqu'à des valeurs de $100 \mathrm{keV} / \mu \mathrm{m}$, puis diminue rapidement pour des TEL plus élevés. Cela veut dire que l'EBR atteint une valeur maximale pour une énergie des neutrons d'environ $0,5 \mathrm{MeV}$ que ce soit pour les cellules végétales ou animales. Des neutrons de $0,4 \mathrm{MeV}$ ont un EBR quatre fois supérieur aux neutrons de $10 \mathrm{MeV}$ (Tubiana et al., 1986).

Après irradiation par des rayonnements à TEL très élevé, ainsi que nous l'avons mentionné plus haut, on observe des courbes de survie exponentielles. Les lésions létales d'emblée augmentent avec le TEL entre 10 et $100 \mathrm{keV} / \mu \mathrm{m}$, puis diminuent pour des TEL > $200 \mathrm{keV}$ (Fig. 9). Dans les mêmes conditions, les lésions potentiellement létales et sublétales suivent une progression parallèle, mais pour une efficacité relative beaucoup moindre. Ceci peut être expliqué par la présence de lésions multiples dues aux dépôts d'énergie de plus en plus importants, qui vont entraîner une mort cellulaire directe. Cet effet est largement indépendant du débit de dose et du fractionnement. Néanmoins une certaine réparation s'effectue tout de même, mais elle est plus lente et donc plus difficile. En conséquence, même pour de faibles doses de neutrons, une partie des lésions ne peuvent être réparées. À l'inverse, un accroissement des dépôts d'énergie - et donc des dommages à la cellule - n'augmentera pas pour autant l'effet des lésions directement létales. La diminution de l'EBR consécutive traduit ce gaspillage d'énergie (« overkill effect »).

\subsection{Importance de l'effet oxygène}

Pour les rayonnements de faible TEL, les doses nécessaires pour produire un effet biologique donné sur un tissu sont 2,5 à 3 fois plus importantes quand le tissu est appauvri en oxygène. Ce phénomène doit être pris en compte pour le traitement de tumeurs, car on sait que les cellules situées à l'intérieur de la masse tumorale sont moins irriguées qu'en périphérie, ce qui entraîne une radiorésistance locale. Cet effet oxygène est plus réduit pour les neutrons (facteur 1,8). Les EBR sont donc plus élevées, en condition hypoxique, pour les rayonnements de faible TEL que pour les neutrons. En effet, la sous-oxygénation inhibe certaines réactions chimiques, diminuant l'effet indirect (radiolyse de l'eau) en proportion. Cette nouvelle répartition du dépôt d'énergie est évidemment moins sensible pour les faibles TEL que pour les TEL élevés, ce qui explique un effet oxygène moins marqué pour les neutrons (Barendsen, 1997).

\subsection{Irradiation neutronique et transformation néoplasique}

La connaissance de la probabilité du risque de cancer induit par les radiations est venue par l'établissement des relations dose-effet. L'hypothèse de travail est, et reste, 
Ph. VOISIN

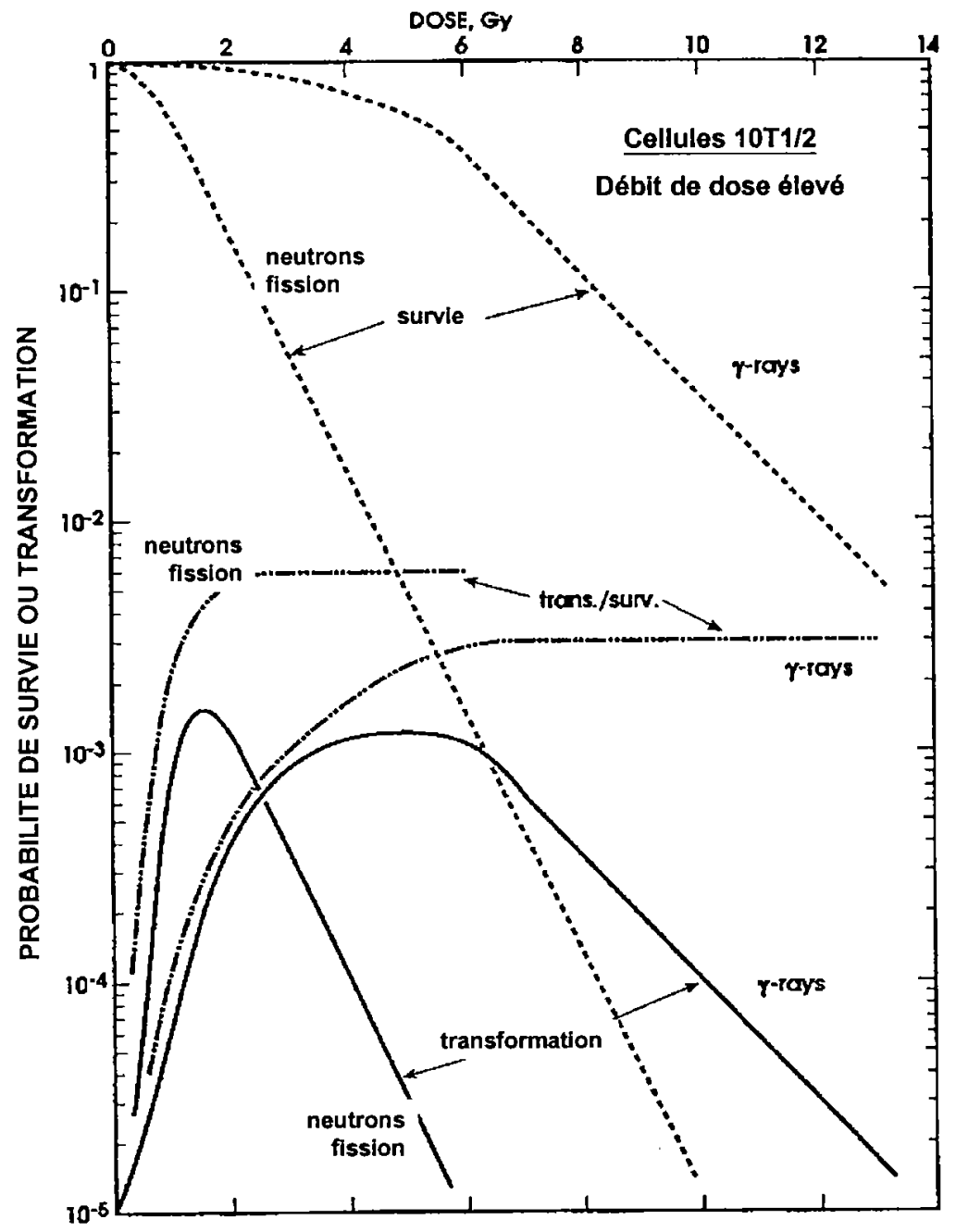

Fig. 10. - Mesure expérimentale de la probabilité de survie ou de la cinétique de transformation néoplasique de cellules de mammiferes exposées soit au rayonnement $\gamma d{ }^{60}{ }^{6} \mathrm{Co}$, soit à des neutrons de fission (D'après Elkind (1997)).

Experimental measurement of the probability of survival or the kinetic of neoplasic transformation of irradiated mammals cells, exposed either to $\gamma$-rays of ${ }^{60} \mathrm{Co}$, or to fission neutrons (from Elkind (1997)). 
que le degré de risque peut être quantifié en termes dose-effet à partir de simples expositions à haut débit de dose, comme à Hiroshima et Nagasaki.

Quand les cellules sont fortement irradiées durant des temps très courts, l'expression de la transformation néoplasique radio-induite due à la dose devient de plus en plus compétitive par rapport à la mort cellulaire (Fig. 10). Classiquement, la pente initiale des courbes de survie est plus grande pour les neutrons que pour les rayonnements de plus faible TEL. La fréquence de transformation néoplasique augmente rapidement au-dessus de la fréquence spontanée de $10^{-5}$, mais la mort cellulaire, augmentant également, fait que la fréquence de transformation se stabilise autour de $10^{-2}$. Cet effet est suivi par une réduction de la fréquence parallèle à l'augmentation de la mort cellulaire. Pour examiner l'influence de la réparation cellulaire, le fractionnement de dose a été utilisé. Le taux de survie augmente alors que le taux de transformation diminue. Si on examine maintenant les effets d'une irradiation prolongée, le taux de survie pour les neutrons ne change pas mais le taux de transformation augmente dans la région des basses doses (jusqu'à 1,5 Gy).

En reprenant la notion de « réparation » cellulaire, après exposition prolongée à un rayonnement de faible TEL, la réparation des lésions sublétales permet de diminuer le taux de mort cellulaire ainsi que le taux de transformation néoplasique. La réparation étant moins effective avec les rayonnements de fort TEL, le taux de survie devient alors indépendant du débit de dose. C'est ce qui se produit pour les neutrons de fission (Elkind, 1997).

\subsection{Radiothérapie par effet neutronique}

Les différents phénomènes présentés ci-dessus montre qu'il y a un grand intérêt à utiliser les neutrons pour traiter les tissus tumoraux. En particulier :

- l'absence relative d'effet oxygène ;

- l'effet moins important du fractionnement de la dose, en terme d'efficacité thérapeutique, de l'irradiation neutronique par rapport à l'utilisation des rayonnements de faible TEL. On peut traduire ce phénomène comme un nivellement de la radiosensibilité individuelle des tissus ;

- par contre, suite à une induction plus grande de transformation néoplasique par les neutrons, il paraît important de concentrer la dose appliquée en un temps court afin d'atteindre plus complètement les tumeurs à prolifération rapide (Tubiana et al., 1986).

La radiothérapie par neutrons rapides est à ce jour réalisée dans au moins 18 centres répartis dans le monde et a touché depuis 50 ans plus de 15000 patients. Cette radio- 
Ph. VOISIN

thérapie est effectuée soit uniquement avec des neutrons, soit en association avec d'autres rayonnements ou avec la chimiothérapie. Elle a amplement montré son efficacité en termes de survie et de contrôle des cancers pour des catégories spécifiques de malades et de pathologies. Il est intéressant de noter qu'il y a là correspondance entre la théorie radiobiologique et l'application clinique. Cependant, les équipements restent onéreux. Les conditions méthodologiques sont toujours améliorées pour diminuer les effets secondaires et augmenter l'efficacité du traitement. Ceci est particulièrement vrai pour le traitement des tumeurs au niveau du système nerveux central. Justement en raison de cette limitation majeure, de nouvelles techniques ont été récemment proposées, et notamment une thérapie par capture neutronique. Un composé boré est incorporé au niveau des cellules cancéreuses. Une activation par neutrons thermiques conduit ensuite à l'émission de particules à TEL élevé selon la réaction ${ }^{10} \mathrm{~B}+\mathrm{n}$ (thermiques) $\rightarrow{ }^{7} \mathrm{Li}+\mathrm{a}+\gamma(0,48 \mathrm{MeV})+2,31 \mathrm{MeV}$. On réalise ainsi une irradiation localisée des cellules ayant fixé les atomes de bore. Des études in vitro ont montré que l'exposition de tumeurs contenant de 100 à $500 \mu \mathrm{g}$ de ${ }^{10} \mathrm{Bg}^{-1}$ à une dose de 2 à 10 Gy de neutrons résulte en une augmentation de la mort cellulaire de 10 à 100 fois par rapport à un traitement de neutrons seuls. Les résultats cliniques obtenus après le traitement in vivo de tumeurs cérébrales inopérables sont encourageants en terme d'efficacité et de survie à long terme. Il reste cependant à améliorer la spécificité de la fixation des atomes de bore sur les cellules-cible.

\section{Conclusion}

Il n'était pas possible dans ce rappel sur les effets biologiques des neutrons de balayer l'ensemble des données expérimentales disponibles ni de décrire toutes les applications théoriques et pratiques s'y référant. Les effets des neutrons sont globalement plus graves que ceux provoqués par les rayonnements de plus faible TEL (Rayonnements $X$ et $\gamma$ ) à tous les niveaux de mesure utilisés, que ce soit les aberrations chromosomiques, la survie cellulaire ou la carcinogénèse. Cette différence est vraisemblablement liée à la densité du dépôt d'énergie dans les cibles vitales de la cellule, et à l'absence de variations significatives liées à l'oxygénation, au débit de dose ou au fractionnement. De tels effets délétères à considérer en cas d'accident de criticité peuvent paradoxalement devenir des avantages en cas d'irradiation thérapeutique.

\section{RÉFÉRENCES}

AIEA (Agence Internationale de l'Energie Atomique) (1986) Biological dosimetry : chromosomal aberration analysis for dose assessment, Technical report series $n^{\circ} 260$.

Alberts B., Bray D., Lewis J., Raff M., Roberts K., Watson J.D. (1990) Biologie moléculaire de la cellule. Flarnmarion, Paris. 


\section{EFFETS BIOLOGIQUES DES NEUTRONS}

Barendsen G.W. (1997) Parameters of linear-quadratic radiation dose-effect relationships : dependence on LET and mechanisms of reproductive cell death. Int. J. Radiat. Biol., 71, 649-655.

Bender M.A., Awa A.A., Brooks A.L., Evans H.J., Groer P.G., Littlefield L.G., Pereira C., Preston R.J., Wachholz B.W. (1988) Current status of cytogenetic procedures to detect and quantify previous exposures to radiation. Mutation. Res, 196, 103-159.

Doloy M.-T. (1991) Dosimétrie basée sur le dénombrement des anomalies chromosomiques contenues dans les lymphocytes sanguins. Radioprotection, 26, suppl. $\mathrm{n}^{\circ} 1,171-184$.

Edwards A.A. (1990) Dosimetric and statistical aspects of cytogenetics. Dans : I reunion international sobre dosimetria biologica (R.H. Crespo, Ed.), pp. 76-85. Lisse, Madrid.

Elkind M.M. (1997) Cell-cycle sensitivity, recovery from radiation damage and an new paradigm for risk assessment. Int. J. Radiat. Biol. 71, 657-665.

Galle P., Paulin R. (1992) Biophysique. I. Radiobiologie - Radiopathologie, pp. 49-68. Masson, Paris.

Geacintov N.E., Swenberg C.E. (1991) Chemical, molecular biology, and genetic techniques for correlating DNA base damage induced by ionizing radiation with biological end points. Dans : Physical and chemical mechanisms in molecular radiation biology (Glass W.A., Varma M.N., Eds), pp.453474. Plenum Press, New York.

Lett J.T. (1992) Damage to cellular DNA from particulate radiations, the efficacy of its processing and the radiosensitivity of mammalian cells. Radiat. Environ. Biophys., 31, 257-277.

Lloyd D.C. (1990) Biological dosimetry by cytogenetic methods. Dans : I reunion international sobre dosimetria biologica, (R.H. Crespo, Ed.) pp. 60-73. Lisse, Madrid.

Poretti G.G. (1988) Abrégé de biophysique des radiations. Presses Polytechniques Romandes, Lausanne.

Preston R.J. (1990) Biological dosimetry: mechanistic concepts. Dans : I reunion international sobre dosimetria biologica, (R.H. Crespo, Ed.) pp. 21-34. Lisse, Madrid.

Tubiana M., Dutreix J., Wambersie A. (1986) Effets des rayonnements sur les molécules d'ADN et les chromosomes. Dans : Radiobiologie, pp. 33-7. Hermann, Paris.

Voisin P., Lloyd D., Edwards A. (1997) Chromosome aberrations scoring for biological dosimetry in a criticality accident. Radiat. Prot. Dosim. 70, 467-470. 